\title{
SPATIAL AND FUNCTIONAL PROPERTIES OF PSEUDO- ALLELES AT THE WHITE LOCUS IN DROSOPHILA MELANOGASTER
}

\author{
M. M. GREEN \\ Department of Genetics, University of California, \\ Davis, California
}

Received 6.viii. $5^{8}$

\section{INTRODUCTION}

THE apparent widespread occurrence of pseudoallelism has called for a re-examination of the gene concept. The need for re-examination comes primarily from seemingly conflicting results derived from independent operational procedures which describe the allelic relationships of separately occurring mutations. Thus two independent mutants are regarded as pseudoalleles if, when compounded, they produce a mutant phenotype and if single crossing-over occurs between them such that two exceptional types of gametes, one carrying neither mutant, the other both mutants, are produced. There is an added phenotypic exception concomitant to pseudoallelism : repulsion compounds of independent recessive mutants manifest a mutant phenotype, coupling compounds of the same mutants are wild-type.

On the one hand the phenotypic observation supports the conclusion that the mutants occupy identical loci, on the other hand the occurrence of crossing-over shows that they are spatially separate. The polemic incident to these observations stems from interpretations which are designed in part to reconcile the conflicts noted above. (Compare Goldschmidt, I955; Green, I955 ; Lewis, 195I, 1955; Pontecorvo, 1952, 1955.) It has been argued that the results of pseudoallelism can be interpreted best if the concept of the gene as one indivisible recombinational, functional and mutational unit is discarded. In its place is substituted a gene constituted of a number of mutational sites, functionally identical yet separable one from the other by crossing-over. This interpretation permits a formal explanation of the phenotypic and recombinational observations associated with pseudoalleles. What remains in doubt, however, is whether this concept of a large overriding functional unit within which crossing-over occurs fits all the facts and is supported by critical experimental test.

When applied to certain instances of pseudoallelism (e.g. the lozenge mutants of Drosophila or the biotinless mutants of Aspergillus) the concept of the overriding functional gene appears to explain all facts submitted to date. In these and similar cases of pseudoallelism all independent mutants produce either closely related or identical phenotypes, regardless of their spatial position defined by crossing-over. 
Presumably since the several separable sites cannot be discriminated by any objective phenotypic criteria, it follows that the sites must be functionally identical, otherwise how could compounds produce mutant phenotypes? This may be the case for pseudoallelic arrays such as lozenge and biotinless but it is all-important to recognise that it is not universally true, and such cases of pseudoallelism as the Star-asteroid (Lewis, 1945) and the bithorax-bithoraxoid (Lewis, 1952, 1955) arrays in Drosophila provide all-important exceptions. In both instances the mutants assigned to separate sites on the basis of crossing-over can be objectively discriminated from each other on phenotypic grounds. If phenotypes are equatable to function, it follows that the pseudoalleles of the Star-asteroid and bithorax-bithoraxoid arrays are functionally as well as recombinationally distinctive. As such they do not support the concept of the overriding functional gene.

Proceeding from these considerations, the question may be asked whether it is possible to make a functional discrimination precisely paralleling spatial disposition among pseudoalleles which lack obvious phenotypic differences. The absence of non-identical phenotypes does not a priori mean that such spatially separate mutants are functionally identical. Many examples can be cited where non-allelic mutants produce inseparable phenotypes through alterations in different steps of biosynthetic pathways. It is therefore not inconceivable that among pseudoallelic mutants a similar situation exists and, despite the phenotypic situation, pseudoalleles govern related, but none the less separate functional steps in the production of inseparable phenotypes.

The rationale underlying the following genetic analysis may be stated as follows. In the absence of specific information on the mechanism of gene action, an indirect approach must be attempted: By subjecting individuals of different genotypes to controlled alterations in their genetic and non-genetic environments it is hoped that measurable phenotypic differences will be uncovered which permit an unambiguous separation of the mutants. If an adequate sample of independent mutants is studied, and if an exact separation can be made, such that absolute agreement between phenotypic and recombinational criteria occurs, the results then parallel the Star-asteroid and bithoraxbithoraxoid pseudoalleles and the need for the concept of separate functional and spatial genes becomes dubious.

The white (w) locus in Drosophila melanogaster was selected for study because it possesses a number of attributes which make it favourable for a detailed study of the functional and spatial propertics of pseudoalleles. Recombination between mutants has been unequivocally demonstratcd (Lewis, 1952, MacKendrick and Pontecorvo, 1952). In addition a number of different genetic factors, which alter the phenotypcs of white pseudoalleles, have been and will be described and these are the basis for tests of function among the pseudoalleles. 


\section{MATERIALS AND METHODS}

(i) Nomenclature and origin of mutants. A total of $36 w$ mutants is included in this report. Mutant symbols used follow the scheme listed in Bridges and Brehme (1944) with certain minor changes. For simplicity all pigmented mutants will be referred to by their superscript notation. Thus apricot $\left(w^{a}\right)$ becomes $a$, apricot-2 $\left(w^{a 2}\right) a^{2}$, cherry $\left(w^{c h}\right)$, ch, etc. All white-eyed mutants are designated in terms of their origin. Thus spontaneous mutants are designated $s-1, s-2$, etc., while X-ray induced mutants are indicated by $x-1, x-2$, etc. The symbol $w$ is reserved for the original white-eyed mutant and for referring collectively to the mutants. With but three exceptions the pigmented mutants apparently are all spontaneous in origin. The mutant col occurred following $\mathrm{X}$-irradiation while both $d p$ and $d p 2$ arose following chlorethyl methane sulphate treatment.

(ii) Design of crossing-over studies. At the outset, it was found that crossing-over occurs between the mutants $a$ and $c h$. From $\$ \& a / c h$ carrying in addition the closely linked marker genes $y$ (yellow body, map position o) and $s p l$ (split eye, $3 \cdot 0$ ) localised to the left and right of $w$, two exceptional recombination classes ( $w^{+}$and $a$ ch coupled to same X-chromosome and white-eyed in phenotype and designated " $w$ ") were found. The results are included in table 2. The crossing-over data show that $a$ is localised to the left of $c h$; therefore these mutants were adopted for use in testing other $w$ mutants. Tester stocks carrying $a$ or $c h$ together with marker genes $y$ and $s p l$ and the II and III chromosome inversions $C y$ (Curly wing) and Ubx (Ultrabithorax-130) balanced to $X a$ (Xasta) were constituted as follows : $y^{2} s u$-r $w^{a} a s p l$; $C y$; $U b x / X a$ and $y$ ac ch spl; $C y$; Ubx/Xa. Heterozygous autosomal inversions were routinely incorporated into these tests since they strikingly increase the crossing-over frequency in the intervals studied and presumably increase recombination between pseudoalleles. The crossing-over percentages for the $y$-w and $w$-spl intervals in the absence of autosomal inversion were found to be $\mathrm{I} \cdot 05$ and 0.37 respectively, while in the presence of both $C y$ and $U b x$ the percentages increased to 4.63 and I .87 .

The linkage relation of each mutant was made by crossing mutant $\delta^{*} \sigma^{*}$ to one

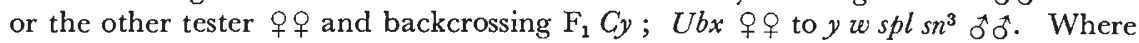
pigmented mutants were tested both $w^{+}$and double mutant recombinants, the latter without exception white-eyed and designated " $w$ ", were scored. Where pure white mutants were tested only the $w^{+}$exceptions were sought since presumably the double mutants are white-eyed.

(iii) Types of phenotypic modifications and their analysis. In principle all $w$ mutants were, prior to crossing-over analysis, subjected to classification based on their reactions in six different phenotypic categories. These are the following :

(I) Dosage compensation vs. non-compensation: This can be scored simply by comparing the phenotypes of $\delta^{*} \sigma^{*} \mathrm{vs}$. 우우. Non-compensation is indicated when 우우 are clearly darker (i.e. nearer wild-type) than $\sigma^{t} \delta$. In table $\mathrm{I}$, the + means compensation, the o non-compensation.

(2) Suppressor of $w^{a}\left(s u-w^{a}\right)$ : A recessive, sex-linked mutant tightly linked to $y$ was found by Schultz to modify the phenotype of $a$ flies toward wild-type. In tests reported here a spontaneous allele of the $s u-w^{a}$ was used. Mutants were tested by crossing to $y^{2} s u-w^{a}$ flies and comparing among the $\mathrm{F}_{2}$ progeny the $y^{2}$ and $y^{+}$mutant flies. The former carry $s u-w^{a}$. In table $\mathrm{I}$, the + indicates suppression, a nonsuppression.

(3) Suppressor of forked (su-f) : By chance it was found that the recessive sexlinked su-f acts as an enhancer of $a$ such that flies of the genotype $a$ su- $f$ are nearly white-eyed. Mutants carrying also $f^{1}$ were tested for the effect of $s u-f$ by crossing to $f^{1}$ su-f followed by comparing among the $\mathrm{F}_{2}$ progeny the phenotypes of forked and suppressed forked mutant $\sigma^{t}$. In table I the + means enhanced by $s u-f$, o noenhancement.

(4) Enhancer of eosin $\left(e n-w^{e}\right)$ : The origin of this sex-linked recessive modifier of $e$ with map location of $32+$ has been reported elsewhere (Green, 1957). The 
associated plcxate-like wing venation is used to identify phenotypically $e n$-re $e^{e}$ which is without effect on eye colour except in association with certain w mutants. Tests of enhancement were made by crossing mutants to $e n-w e$ followed by comparing among the $\mathrm{F}_{2} w$ mutant ot progeny those with and without the enhancer. In table 1 the + signifies enhancement and o non-enhancement of the cye colour.

TABLE 1

Summary of phenotypic interactions of $\mathrm{w}$ mutants

\begin{tabular}{|c|c|c|c|c|c|c|}
\hline $\begin{array}{c}w \\
\text { mutant }\end{array}$ & $\begin{array}{c}\text { Dosage } \\
\text { compensation }\end{array}$ & $s u-w^{a}$ & $s u-f$ & $s u-z$ & $e n-w^{e}$ & $\begin{array}{l}\text { Interaction } \\
r b \text { and } g\end{array}$ \\
\hline$a$ & + & + & + & 0 & 0 & + \\
\hline$a_{2}$ & + & o & o & 0 & 0 & 0 \\
\hline$a_{3}$ & + & o & 0 & o & 0 & 0 \\
\hline$a_{4}$ & + & 0 & 0 & o & 0 & + \\
\hline$a E$ & 0 & $\ldots$ & $\ldots$ & + & + & $\ldots$ \\
\hline$b f$ & + & o & 0 & 0 & 0 & + \\
\hline $\begin{array}{l}o f \\
b l\end{array}$ & + & $\cdots$ & $\cdots$ & 0 & $\cdots$ & $\cdots$ \\
\hline co & $\begin{array}{l}+ \\
+\end{array}$ & ${ }^{\prime \prime}$ & $\ddot{0}$ & $\begin{array}{l}0 \\
0\end{array}$ & $\begin{array}{c}\cdots \\
0\end{array}$ & $\ddot{t}$ \\
\hline col & + & o & 0 & o & 0 & + \\
\hline$c h$ & 0 & 0 & 0 & + & + & + \\
\hline$c h^{2}$ & + & $\ldots$ & $\ldots$ & o & 0 & $\ldots$ \\
\hline$d p$ & + & $\cdots$ & $\cdots$ & o & 0 & $\ldots$ \\
\hline$d_{p} 2$ & $+_{0}^{+}$ & $\cdots$ & $\cdots$ & 0 & o & $\ddot{+}$ \\
\hline $\begin{array}{l}e \\
e 2\end{array}$ & $\begin{array}{l}\text { o } \\
\text { o }\end{array}$ & $\begin{array}{l}0 \\
0\end{array}$ & $\begin{array}{l}0 \\
0\end{array}$ & + & $\begin{array}{l}+ \\
+\end{array}$ & $\begin{array}{l}+ \\
+\end{array}$ \\
\hline $\begin{array}{l}e 2 \\
e c 2\end{array}$ & + & 0 & 0 & 0 & o & + \\
\hline$h$ & + & 0 & o & + & + & $\ldots$ \\
\hline$i$ & 0 & $\ldots$ & $\ldots$ & 0 & $\ldots$ & $\ldots$ \\
\hline sat & + & o & 0 & 0 & 0 & + \\
\hline$s p-w$ & + & $\ldots$ & $\ldots$ & + & $\ldots$ & $\ldots$ \\
\hline$w$ & $\ldots$ & o & 0 & + & $\ldots$ & $\cdots$ \\
\hline$s-1$ & $\ldots$ & 0 & $\ldots$ & o & $\ldots$ & $\ldots$ \\
\hline$s-2$ & $\ldots$ & $\ldots$ & $\ldots$ & 0 & $\ldots$ & $\ldots$ \\
\hline$s-3$ & $\ldots$ & $\ldots$ & $\ldots$ & 0 & $\ldots$ & $\ldots$ \\
\hline$s-4$ & $\ldots$ & 0 & $\ldots$ & 0 & $\ldots$ & $\ldots$ \\
\hline$s-5$ & $\ldots$ & $\ldots$ & $\ldots$ & o & $\ldots$ & $\ldots$ \\
\hline$s-9$ & $\ldots$ & $\ldots$ & $\ldots$ & o & $\ldots$ & $\ldots$ \\
\hline$s=10$ & $\cdots$ & 0 & $\cdots$ & 0 & $\cdots$ & $\ldots$ \\
\hline$x-1$ & $\ldots$ & $\ldots$ & $\ldots$ & 0 & $\ldots$ & $\ldots$ \\
\hline$x-2$ & $\ldots$ & $\ldots$ & $\ldots$ & 0 & $\ldots$ & $\ldots$ \\
\hline$x-3$ & $\ldots$ & $\cdots$ & $\ldots$ & 0 & $\ldots$ & $\ldots$ \\
\hline$x-4$ & $\ldots$ & $\ldots$ & $\ldots$ & o & $\cdots$ & $\cdots$ \\
\hline$x-5$ & $\cdots$ & $\ldots$ & $\cdots$ & 0 & $\ldots$ & $\ldots$ \\
\hline $\begin{array}{l}x-\overline{6} \\
x-8\end{array}$ & $\cdots$ & 0 & $\cdots$ & o & $\cdots$ & $\cdots$ \\
\hline $\begin{array}{l}x=8 \\
x-16\end{array}$ & $\begin{array}{l}\cdots \\
\cdots\end{array}$ & $\begin{array}{c}\cdots \\
\mathrm{o}\end{array}$ & $\begin{array}{l}\cdots \\
\ldots\end{array}$ & $\begin{array}{l}0 \\
+\end{array}$ & $\begin{array}{l}\cdots \\
\cdots\end{array}$ & $\cdots$ \\
\hline
\end{tabular}

(5) Interaction with ruby $(r b)$ and garnet $(g)$ : It has been long known that certain pigmented $w$ mutants interact with non-allelic eye colour mutants to produce a new phenotype lighter than the phenotype associatcd with either mutant (Morgan and Bridges, 1913). Thus $a r b$ or $a g$ flies are nearly white-eyed. Tests for interaction with $r b$ and $g$ were made by the usual methods utilising crossing-over to obtain the coupling combinations. In tablc $\mathrm{I}$ the + mcans the mutant intcracts with $r b$ and/or $g$, the o means no interaction with the phenotype inseparable from that of $r b$ or $g$. A detailed study of this situation will be presented elsewhere.

(6) Suppression of zeste (su-z) : In the course of analysing the cytogenetics of the sex-linked recessive $z$ mutant of D. melanogaster, Gans (r953) made the interesting observations that in + 우 homozygous for $z$ and heterozygous for $w$ mutants, some $w$ mutants act as dominant suppressors of $z$ while other $w$ mutants do not. 
Thus, depending on the $w$ mutant, 우 of the genotype $z w^{+} / z w$ are either zeste or near wild-type in phenotype. Mutants were tested for suppression of $z$ by obtaining $\delta^{*} \hat{O}^{*} z$ and scoring the phenotype of their $q$ progeny after crossing to $z w^{+}$웅.

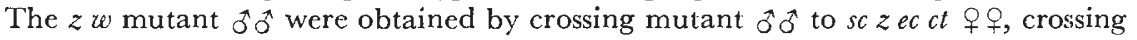
the $\mathrm{F}_{1}$ inter se and selecting $\mathrm{F}_{2}$ ô $s c z w$. Where a pigmented $w$ mutant was tested, sc $z w$ ô $\hat{\sigma}^{t}$ were readily identified since $z$ dilutes the mutant eye colour. No such identification could be made when a pure white-eyed mutant was used. Rather

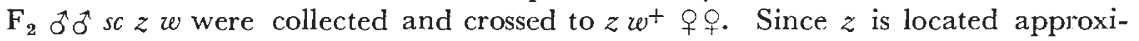
mately equidistant between $s c$ and $w$, half the $s c w \mathrm{~F}_{2} o^{t} o^{t}$ derived from 우오 sc zec ct/w should be sc $z w$, half sc $z w^{+}$. Routinely $1_{2} \mathrm{~F}_{2}$ sc w ô $\hat{o}^{+}$were tested to assure the recovery of at least one sc $z w d$. In table I the + indicates suppression of zeste and the o non-suppression.

\section{EXPERIMENTAL RESULTS}

The results of the several phenotypic tests with the various $w$ mutants are listed in table $I$ and are for the most part self-explanatory. A few comments are, however, in order. The action of the su-wa and su-f appears to be specific for $a$ and as such permits no discrimination among the $w$ mutants. Of interest here are observations made with several mutants derived from $a$ and tested to the $s u-w^{a}$ and $s u-f$. Two partial back mutations of $a$ proved upon analysis to be distinctive : one is suppressed by $s u-w^{a}$ and enhanced by $s u-f$; the second is unchanged by either suppressor mutant. On three separate occasions $a$ has mutated spontaneously to a state no longer suppressible although producing a phenotype in the absence of $s u$ - $w^{a}$ inseparable from that of $a$ itself. This is not unlike the situation already described for the forked $(f)$ locus in Drosophila where by means of X-irradiation it has been possible to mutate the suppressible $f^{1}$ mutant to an unsuppressible state (Green, I956).

Among the tests applied, three, dosage compensation, $e n-w^{e}$ and suppression of $z$, appear promising since they discriminate among several mutants. A comparison of the tests among pigmented mutants shows that with two exceptions they are in agreement. Thus all dosage compensating mutants, with the exception of $h$, are unaltered by $e n-w^{e}$ and do not act as suppressors of $z$. Similarly with the exception of $i$ all non-compensated mutants are enhanced by $e n-w^{e}$ and function as dominant suppressors of $z$.

Within the group of white-eyed mutants discrimination can be made only in their behaviour toward $z$.

With the completion of the several phenotypic tests each mutant was tested for recombination to either the $a$ or $c h$ tester. Since the behaviour toward $z$ provided the widest discrimination among the mutants, it was adopted as a guide for crossing-over tests. Initially all $z$ suppressors were tested to $a$ and all non-suppressors to $c h$.

The outcome of crossing-over tests between $z$ suppressing mutants and $a$ are listed in table 2. The results are self-explanatory. Wildtype exceptions arising in association with crossing-over were recovered in tests with each mutant. Among the pigmented mutants tested, 
white-eyed exceptions which comprise the double mutant class were similarly recovered as reciprocals of the $w^{+}$exceptions. On the basis of the distribution of the marker genes among the exceptions, each of the $z$ suppressing mutants must be localised to the right of $a$. Presumed double mutants in all cases suppress $z$. The recombination relations of three other $z$ suppressing mutants listed in table I have alrcady been determined. Lewis (I956) has shown that $w$ and $s p$-w are localised to the right of $a$ and MacKendrick (1953) has localised $a E$ to the right of $c o$ which, as will be noted below, is a putative allele of $a$.

All non-suppressors of $z$ were testcd for recombination with ch and the results are tabulated in table 3. In cach instance, with the

TABLE 2

Crossing-over between a and $\mathrm{w}$ mutants which suppress $\mathrm{z}$

\begin{tabular}{|l|c|c|c|}
\hline $\begin{array}{c}\text { w mutant } \\
\text { tested }\end{array}$ & \multicolumn{2}{|c|}{ Total recombinants } & $\begin{array}{c}\text { Total } \\
\text { progeny }\end{array}$ \\
& $y^{2}$ " $w "$ & $w^{+} s p l$ & \\
& & & \\
$c h$ & 1 & 2 & 32,466 \\
$e$ & 3 & 1 & 21,053 \\
$e 2$ & 2 & 2 & 32,466 \\
$h$ & 2 & 2 & 28,896 \\
$x-16$ & 0 & 2 & 30,575 \\
\hline
\end{tabular}

exception of $b f, w^{+}$individuals associated with crossing-over were found. Among pigmented mutants, including $b f$, white-eyed reciprocal exceptions were recovered as well.

That the white-cyed exceptions represent the double mutant class is demonstrated by these facts. First, the white-eyed genotype suppresses $z$ and second, from $q$ q 9 of the presumed genotype $y^{2} s u-w^{a} a c h /+\times$ $y w s p l s n^{3} \delta^{\lambda} \delta^{\lambda}$, I $\circ y^{2} s u-w^{a} a \mid y w s p l s n^{3}$ was recovered among 25,799 progeny. Similarly among 39,300 progeny of $+9+$ presumed to be $a^{3} c h s p l l+\times y w s p l s n^{3} \hat{o}$, one $a^{3} \hat{\jmath}$ was found. Thus all mutants gave exceptions which, based upon the distribution of the marker genes, show that they are, without exception, localised to the left of $c h$. In addition to the mutants of table 3 , three additional non- $z$ suppressing mutants, $b l$, co and $i$ have been found by MacKendrick (I953) to be localised to the left of $c h$.

The data can be summarised as follows : among the $3^{6}$ mutants studied by Lewis, by MacKendrick and here, the $8 z$-suppressing mutants are localised to the right of $a$ and the 28 non- $z$ suppressing mutants are localised to the left of $c h$. There are no overlaps.

In the several instances attributed to pseudoallelism in microorganisms the analysis of recombination among numerous independent mutants is consistent with the interpretation that the mutants are spatially separate from one another, i.e. they are continuously distributed along the chromosome (Benzer, r957; Demerec et al., I956 ; 
Pontecorvo, I955; Streisinger, and Franklin, 1956). This situation finds no counterpart in Drosophila although admittedly with the exception of the lozenge (Green and Green, 1956) and singed (Hexter, I957) pseudoalleles an insufficient number of mutants have been studied. Since phenotypic scoring in the case of $w$ mutants can be made with relative ease, extensive tests were undertaken to determine whether additional loci could be uncovered among a selected group of non- $z$ suppressing $w$ mutants. Several mutants were tested for

TABLE 3

Crossing-over tests between $\mathrm{ch}$ and $\mathrm{w}$ mutants all of which fail to suppress $\mathrm{z}$

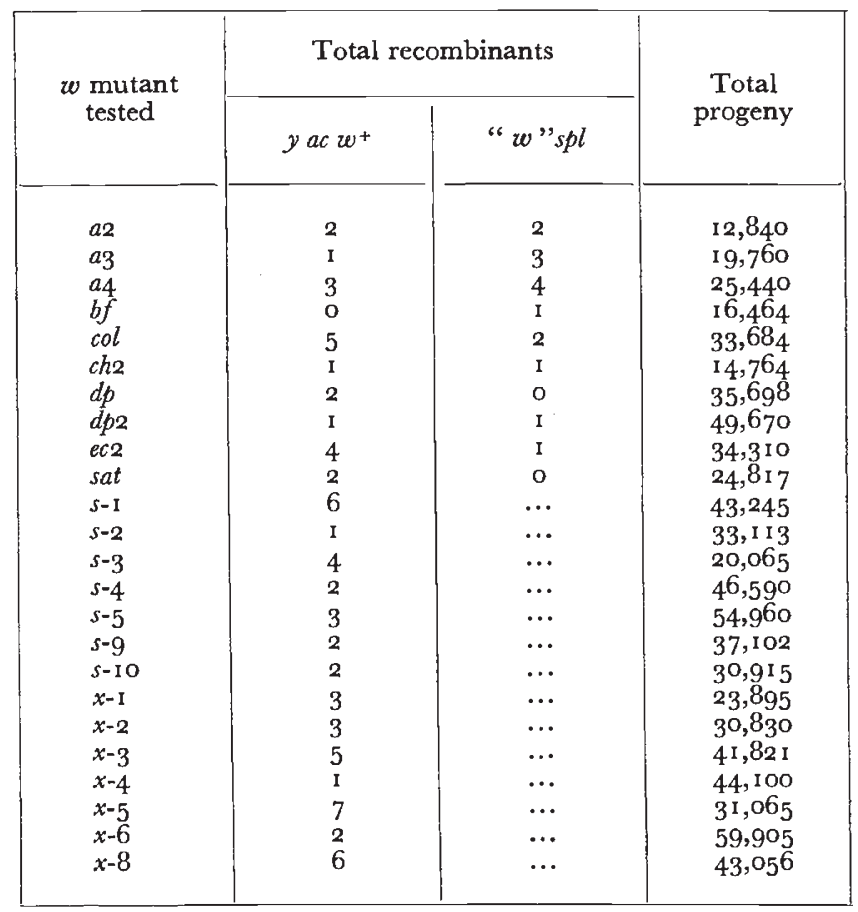

recombination with either $a, a_{2}$, or $a_{4}$ or with all. Markers and inversions were included as described earlier. The results of these crosses are tabulated in table 4. Five mutants, $b f$, col, sat, s-4 and $x-6$ gave $w^{+}$ recombinants when tested to either $a$ or $a 2$. In each case the marker associated with the $w^{+}$exception demonstrates that spatially these mutants must all lie to the left of $a$ or $a 2$. From 우 $b f / a$, white-eyed exceptions carrying markers reciprocal to those of the $w^{+}$exception were also found. Presumably these represent the double mutant class. These data, taken together with the report of Lewis (1957) on sp-w, demonstrate the existence of at least four spatially discrete $w$ loci.

The absence of recombinants in the extensive progeny scored from crosses $a_{1} a_{2}, a_{3}, a_{4}$ and $c o$ warrant the conclusion that these mutants are truly allelic and occupy identical loci on the chromosome. In 
summarising these data it should be noted that from $q q a / a_{2}$ something over 400 ,ooo progeny, equivalent to approximately $1 \cdot 6-2 \times 10^{6}$ progeny in the absence of autosomal inversions $C y$ and $U b x$, were scored without detecting recombinant exceptions expected of pseudoalleles. Additional negative results were obtained with $q$ q $a / a_{4}$ where approximately 375 , ooo progeny, equivalent to $1 \cdot 5 \times 10^{6}$ without autosomal inversions, were scored. Collectively among the other crosses yielding negative results (including progeny from o우 $a_{1} / a_{3}, a_{2} / a_{3}, a_{2} / a_{4}$ and $a_{3} / a_{4}$ )

TABLE 4

Crossing-over among $\mathrm{w}$ mutants which fail to suppress $\mathrm{z}$

\begin{tabular}{|c|c|c|c|c|}
\hline \multirow{2}{*}{$\begin{array}{c}\text { Tester } \\
\text { genotype }\end{array}$} & \multirow{2}{*}{$\begin{array}{l}w \text { mutant } \\
\text { tested }\end{array}$} & \multicolumn{2}{|c|}{ Total recombinants } & \multirow{2}{*}{$\begin{array}{c}\text { Total } \\
\text { progeny }\end{array}$} \\
\hline & & $y^{2} w^{+}$ & "w"spl & \\
\hline$y^{2} s u-w^{a} a s p l$ & $\begin{array}{l}a_{2} \\
a_{3} \\
a_{4} \\
b f \\
c o \\
c o l \\
\text { sat } \\
s-4 \\
x-6\end{array}$ & $\begin{array}{c}\cdots \\
\cdots \\
\cdots \\
I \\
\cdots \\
\cdots \\
I \\
4 \\
4\end{array}$ & $\begin{array}{c}* \\
\ldots \\
* \\
2 \\
\ldots \\
\ldots \\
\ldots \\
\ldots \\
\ldots\end{array}$ & $\begin{array}{r}226,7 \text { I } 9 \\
\text { I I I } 934 \\
\text { I } 80,284 \\
80,800 \\
55,275 \\
\text { I 02, I } 45 \\
74,840 \\
\text { I I } 9,435 \\
\text { I I } 3,635\end{array}$ \\
\hline$y^{2} s u-w^{a} a^{2} s p l$ & $\begin{array}{l}a_{3} \\
a R M \\
a R_{57 j} \\
\text { co } \\
\text { col }\end{array}$ & $\begin{array}{l}\ldots \\
\ldots \\
\cdots \\
\cdots \\
2\end{array}$ & $\begin{array}{l}* \\
\ldots \\
\ldots \\
\ldots \\
\ldots\end{array}$ & $\begin{array}{l}96,939 \\
83,412 \\
98,395 \\
89,838 \\
79,485\end{array}$ \\
\hline$y^{2} s u-w^{a} a^{4} s p l$ & $\begin{array}{l}a_{2} \\
a_{3} \\
a R M \\
a R_{57 j} \\
\text { col }\end{array}$ & $\begin{array}{l}\cdots \\
\cdots \\
\cdots \\
\cdots \\
\cdots\end{array}$ & $\begin{array}{l}\cdots \\
\cdots \\
\cdots \\
\cdots \\
\cdots\end{array}$ & $\begin{array}{r}\text { I OO, O I } 5 \\
50,2 \text { I } \\
\text { I 00,926 } \\
93,744 \\
51,255\end{array}$ \\
\hline
\end{tabular}

* See text for explanation.

about 360,000 progeny, equivalent to about $1 \cdot 5 \times 10^{6}$ without inversions, were examincd. (The partial back mutations of $a, a R M$ and $a R_{57 j}$ have been grouped with $a$ in these summarisations.) It should be noted further that these negative rcsults are not confounded by the possibility that the mutants are associated with structural changes such as a short rearrangement or loss which would preclude crossing-over. The fact that recombination occurs betwcen $a$ and four non- $z$ suppressing mutants and between $a 2$ and col, plus the fact that partial or complete back mutations of $a, a_{2}$ and $a_{4}$ have been obtained, militatcs for the absence of associated structural changes.

For the sake of complction it should be pointed out that whilc no evidence for pseudoallelism was uncovered in certain of the aforementioned crosses, two other types of exceptions were found. These 
will be but briefly described here. From $q$ ㅇ $y^{2} s u$-wa $w^{a}$ spl/a4, whiteeyed exceptions carrying the $s p l$ marker have been consistently found in a frequency of about $1 / 10,000$ progeny $(17 / 177,784)$. Although incomplete, analysis of these exceptions suggests that they are possibly associated with a small loss in the $w$ region presumably arising by an altered pairing of $a$ and $a_{4}$ bearing chromosomes. Significantly, the reciprocal recombinant, certainly not $w^{+}$in this case, has defied detection.

From $ㅇ y^{2} s u-w^{a} a s p l / a 2$, white-eyed $q$ ox exceptions carrying the $s p l$ marker have been regularly recovered. Genetic and cytological analysis has shown that these of lethal exceptions are associated with a loss of regions ${ }_{3} \mathrm{~A}_{4-3} \mathrm{C}_{1}$ inclusive of the salivary gland $\mathrm{X}$-chromosome and are presumably the consequence of non-homologous pairing and crossing-over. Identical exceptions have been obtained from $q$ 우 homozygous $a$, homozygous $a_{2}$ and $a_{2} / a_{3}$. The reciprocals, certainly not $w^{+}$, have yet to be detected. The detailed analysis of both types of exceptions is not germane to the considerations here but will be presented, in detail, elsewhere.

Finally, on three separate occasions, $w^{+}$individuals have been found in the absence of crossing-over. One occurred as a single $w^{+} ; U b x /+0^{*}$ from the cross $y^{2} s u-w^{a} a^{4} s p l / a 2 ; C y /+; U b x /+\times y w s p l s n^{3}$. The other two occurred as single $w^{+} \mid y w s p l s n^{3}$ 우 from crosses $y$ ac col $s p l / a 2 \times y w s p l s n^{3}$ and $y^{2} s u-w^{a} a R M s p l / a 2$. These exceptions are interpreted as back mutations of $a 2$.

\section{DISCUSSION}

Before attempting to evaluate the results presented above, it is necessary to make clear what meaning is attached to "gene function" especially as applied to pseudoalleles. Since information on the precise mechanism of gene function is limited, any discussion of gene function must be conceptual, dealing primarily in semantics rather than specific facts.

For any given group of pseudoallelic mutants it cannot be denied that all are affecting the production of a particular phenotype in a similar manner. Specifically for the $w$ pseudoalleles all mutants must be affecting eye colour synthesis similarly. What is of significance here is whether spatially discrete mutants are affecting eye colour synthesis in a similar or identical fashion. For similar functions is understood to mean that mutants are related but none the less divergent in function; identical means the mutants have inseparable function and, in short, are duplicates of each other.

It is quite conceivable that in the case of the $w$ mutants, for example, all spatial loci are functionally integrated to produce a single productperhaps an enzyme-which is prerequisite to normal eye colour synthesis. In this sense all pseudoalleles can be said to have a single 
function, the production of a specific enzyme. However, it is conceivable that each spatial locus contributes to the synthesis of the integrated product in a manner different from its neighbours. Specifically, each spatial locus may be concerned with the incorporation of a different moiety into the integrated whole and the cooperation of all loci necessary before the product can be synthesised. In this meaningand this is the sense of usage here-each locus has a discrete function.

Any concept of the functional identity of pseudoalleles carries the tacit assumption that the spatially separable components are duplicates. A number of facts argue against such a concept. The very fact that mutations of related, recessive mutants can be found demonstrates that the mutants cannot be identical.

Almost without exception, where two pseudoalleles are coupled to the same chromosome, the resultant phenotype is equivalent to and inseparable from that of the most extrcme mutant recognised in the series. As noted above, two coupled $w$ mutants produce a white-eyed phenotype. Similar observations have been noted for the lozenge and forked pseudoalleles. This phenotypic effect is hardly expected under a concept of duplicate functions for those pseudoalleles, e.g. $w$ and $f$, known to be hypomorphs.

Further, certain predictions of the genetic behaviour of pseudoalleles are possible if they are duplicates. Drawing upon experiences with such tandem duplications in Drosophila as Bar (Sturtevant, I925), the Star duplication (Lewis, I94I), and Beadex-recessive (Grcen, I953) it can be concluded that unequal crossing-over is a characteristic common to all. In the case of a number of arrays of pseudoallelic mutants, e.g. white and lozenge, their genetic length is comparable to that of the aforementioned duplications. Accordingly it would be predicted that unequal crossing-over would be a genetic consequence associated with pseudoalleles. Instead pscudoallelic mutants are characterised by the absence of unequal crossing-over, and one of the most consistent features of pseudoalleles seems to be the regular, two by two pairing of such loci. The absences of asymmetrical or non-two by two pairing encountered in tandem duplications can best be attributed to the fact that with pseudoalleles a sufficient degrcc of divergence must cxist among contiguous loci to assure their regular pairing propertics.

Granting the premise that the 36 independent $w$ mutants studied here constitute a fair approximation of the variety of possible mutational types, a number of conclusions are warranted by the data presented. If only the crossing-over information presented here and by others is considered, it is concluded that the w mutants are distributed among four spatial sites. This fact, together with the negative results from extensive intralocus tests, does not support the conclusion of Pontecorvo and Roper (1956) arrived at, in part, from other considerations that " the number of mutational sites within one section of allelism (or gene) separablc from one another by crossing-over may run into high 
numbers". At least for Drosophila the data accumulating from studies of pseudoallelism do not support this view. Needless to say the type and nature of genic organisation peculiar to Drosophilaand presumably for other higher organisms-need not be true for micro-organisms and vice versa. In view of the relatively divergent complexities of cellular organisation and differentiation required by micro-organisms as contrasted to higher forms, it would be surprising indeed to find that they agreed in all details of their genetic organisation.

A comparison between the phenotypic interactions and crossing-over relationships of the $w$ mutants shows that in two respects they are completely correlated. All eight mutants which suppress $z$ have loci to the right of $a$; none of the 28 mutants which fail to suppress $z$ is so located. Classification of the pigmented mutants with $e n-w^{e}$ coincides precisely with the $z$ results. All enhanced mutants occupy loci to the right of $a$; those not enhanced have loci to the left of $c h$. These facts have an all-important bearing on the question of the spatial and functional interrelationships of pseudoalleles.

The experimental data of Gans (1953) are altogether convincing that the $z$ mutant depends upon the mutational and dosage conditions of the $w$ locus for its phenotypic expression. The discovery that the 2 phenotype is suppressed in homozygous 우우 by a single dose of certain $w$ mutants but not by others implies that these mutants differ in some basic functional attribute. Further, the fact that the recessive $w$ mutants act as dominant suppressors suggests that this action is an inherent attribute of these mutants and is without counterpart among other $w$ mutants. It is therefore not unreasonable to assume that for a particular $w$ mutant the condition or substance which determines its eye phenotype is either identical with or related to that which determines its reaction toward $z$. From this assumption, it follows that in the case of two pseudoallelic mutants, each determining a white-eyed phenotype but one suppressing $z$, one not, they differ in the specific alteration producing both phenotypic properties. In short, they are functionally distinctive. Thus the $w$ mutants fall into two groups; the members of each group have spatial and functional attributes which are identical and, as such, each group is an entity different from all other genes. Presumably since there are two spatial sites within each group it should be possible by means of added phenotypic tests to further divide these into discrete functional groups as well. The argument used in connection with the $z$ relationship applies equally well to the $e n-w^{e}$ behaviour and need not be repeated.

The conclusion to be drawn from these considerations is that in the case of pseudoalleles where phenotypic differences are not apparent, objective separations can be made. Thus in principle the $w$ pseudoalleles are identical to Star-asteroid and bithorax-bithoraxoid in that functional and spatial groupings coincide and there is no support for the idea that pseudoalleles can best be represented by a large number of mutational sites functionally identical but separable by crossing-over. 
On the basis of thesc considerations there is no need for two kinds of genes, those of function, those of position. Since, operationally speaking, contiguous pscudoallelic loci are separable in function as well as location they are non-allelic by the same attributes that other genes are non-allelic.

\section{SUMMARY}

r. The phenotypic and crossing-over relationships of 36 independent white mutants in $D$. melanogaster have been investigated.

2. On the basis of thcir interaction with zeste and en-we the mutants can be assigned to two phenotypic groups.

3. The crossing-over studies show that $(a)$ the mutants can be assigned to one of four loci and $(b)$ mutants of one phenotypic group do not spatially overlap those of the sccond group.

4. These findings are interpreted to mean that the mutants of each group are functionally as well as spatially discrete from those of the alternative group. As such the mutants are non-allelic by available operational criteria.

Acknowledgments.-Most of the mutant stocks have been obtained through the generosity of Professor E. B. Lewis, Mme. M. Gans and Dr Jack Schultz to whom I am deeply indebted. Part of this work was done at the Gcnetics Laboratory, University of Leiden, under the auspices of Fulbright and Guggenheim Foundation Fellowships. Thanks are due to Professor H. Gloor for his hospitality and aid.

\section{REFERENCES}

BENZER, s. 1957. The elementary units of heredity, pp. 79-93. The Chemical Basis of Heredity. The Johns Hopkins Press, Baltimore.

BRIDGES, C. B., AND BREHME, K. s. 1944. The mutants of Drosophila melanogaster. Carnegie Inst. Wash. Publ., 552, I-252.

demerec, m., et al. 1956. Genetic studies with bacteria. Carnegie Inst. Wash. Publ., $612, \mathrm{I}-136$.

GANs, M. 1953. Étude génétique et physiologique du mutant $z$ de Drosophila melanogaster. Bull. Biol. France et Belgique, suppl., 38, 1-9o.

Gol.dschmidt, R. B. I955. Theoretical Genetics. University of California Press, Berkeley and Los Angeles.

GREEN, M. M. 1953. The Beadex locus in Drosophila melanogaster: Genetic analysis of the mutant Bx ${ }^{r 49 k}$. Z.I.A.V., 78, 435-449.

green, M. M. 1955. Pseudoallelism and the gene concept. Amer. Nat., 89, 65-7I.

GREEN, M. M. I956. A further analysis of the forked locus in Drosophila melanogaster. P.N.A.S., 42, 73-77.

GREEN, M. M. I957. D.I.S., 3I, 8I .

GREEN, M. M., AND GREEN, K. C. 1956. A cytogenetic analysis of the lozenge pseudoalleles in Drosophila. Z.I.A.V., 87, 708-721.

heXter, w. м. 1957. Genetic resolution in Drosophila. Rec. Genet. Soc. America, 26,376 .

LEWIS, E. B. 1941. Another case of unequal crossing-over in Drosophila melanogaster. P.N.A.S., 27, 31-34.

LEWIS, E. B. 1945. The relation of repeats to position effect in Drosophila melanogaster. Genetics, 30, 137-166.

LEwis, E. B. 1951. Pseudoallelism and gene evolution. Cold Spring Harbor Symp., $16,159^{-1} 74$. 
LEWTS, E. B. 1952. Pseudoallelism of white and apricot in Drosophila melanogaster. P.N.A.S., 38, 953-96ז .

LEWTS, E. B. $\quad$ 1955. Some aspects of position pseudoallelism. Amer. Nat., 89, 73-89. LEWIS, E. B. 1956. An unstable gene in Drosophila melanogaster. Rec. Genet. Soc. America, 25, 65 I.

MACKENDRICK, E. M. I953. D.I.S., 27, IOO.

MACKENDRICK, E. M., AND PONTECORvo, G. 1952. Crossing-over between alleles at the $w$ locus in Drosophila melanogaster. Experientia, 8, 390.

MORGAN, T. H., AND BRIDGES, C. B. I9I3. Dilution effects and bicolorism in certain eye colors of Drosophila. J. Exp. Zool., 15, 429-466.

PONTECORVO, G. 1952. The genetical formulation of gene structure and action. Adv. Enzym., 13, I 2 I-1 49 .

PONTECORvo, G. I955. Gene structure and action in relation to heterosis. Proc. Royal Soc. Lond. B, I44, I 7 I-I 77.

PONTECORVO, G., AND ROPER, J. A. I956. Resolving power of genetic analysis. Nature, $178,83-84$.

STREISINGER, G., AND FRANKLIN, N. C. 1956. Mutation and recombination at the host range genetic region of phage $\mathbf{T}_{2}$. Cold Spring Harbor Symp., 21, I 03-I I I.

STURTEVANT, A. H. I925. The effects of unequal crossing-over at the bar locus in Drosophila. Genetics, 10, I 7-147. 